\title{
Study on the Pathogenesis of Diabetic Nephropathy
}

\author{
Qi Jiang, Yingying Liu* \\ Department of Nephrology, China-Japan Union Hospital of Jilin University, Changchun, Jilin, 130033 \\ ${ }^{*}$ Corresponding author
}

Keywords: Diabetic Nephropathy, Pathogenesis

\begin{abstract}
Diabetes mellitus is caused by a variety of reasons of metabolic disorders characterized by chronic hyperglycemia. Since 1980, the WHO common diagnostic criteria in the world, the American Diabetes Association (ADA) in 1997 proposed the diagnosis of diabetes diagnostic criteria: Symptoms + random glucose $\geq 11.1 \mathrm{mmol} / \mathrm{L}$ (200 mg / dl), or FPG $\geq 7.0 \mathrm{mmol} / \mathrm{L}$ (126 $\mathrm{mg} / \mathrm{dl}$ ), or 2HPG> $11.1 \mathrm{mmol} / \mathrm{L}$ (200 mg / dl) in OGTT. Atypical symptoms are to be confirmed again in another day [1]. Diabetic nephropathy (DN) is one of the important complications of diabetic microangiopathy. This paper collected 60 clinically diagnosed DN analysis, and focuses on its pathogenesis and prevention issues.
\end{abstract}

\section{Introduction}

The occurrence and development of diabetic nephropathy is the result of multifactorial combination of factors, including the disorder of glucose metabolism, changes of renal hemodynamics, a variety of cytokines and genetic background play a very important role. Although the pathogenesis of type I and type II diabetes is different, they are all characterized by persistent hyperglycemia as their basic biochemical characteristics. Therefore, in the pathogenesis of diabetic nephropathy, the impact of hyperglycemia on the kidney has been an important area of research. In the 1993 New England Journal of Medicine, the American Diabetes Association, the Diabetes Control and complicationstrial (DCCT) paper, a landmark in the study of diabetes, was published [N Eng J Med, 1993, 329: 977]. This prospective study of 1,441 patients with type 1 diabetes at 29 medical centers in the United States and Canada over the past 10 years confirms that long-term maintenance of blood glucose at insulin regimens can effectively delay the development of diabetic nephropathy and slow down its progress. Recently, a UK prospective study (UKPDS) of 3867 patients with type 2 diabetes undergoing insulin-intensive therapy was published. After eight years of long-term observation shows that insulin-intensive treatment can also significantly reduce the incidence of type 2 diabetic nephropathy (Lancet, 1998, 352: 837). With convincing clinical research data affirmed the role of hyperglycemia in the occurrence and development of diabetic nephropathy.

\section{Polyol Metabolic Pathway Activation}

Polyol access, also known as sorbitol access. It is composed of aldose reductase (AR) and sorbitol dehydrogenase (SDH). The reaction is as follows: Under high glucose in diabetes, secondary intracellular high glucose can activate key enzyme AR, resulting in a large number of glucose into sorbitol, and sorbitol very strong, can not freely through the cell membrane, so a large number of accumulation in the cell, resulting in hyperosmolar state. A large number of extracellular fluid infiltration, so that cell edema, and ultimately lead to the destruction of cell structure. Sorbitol accumulates to form an osmotic gradient and D-glucose competes with the inositol carrier, whereupon the intracellular pool of inositol is depleted. As inositol is directly involved in phosphatidylinositol synthesis, so that the cell membrane $\mathrm{Na}+-\mathrm{K}+$-ATPase activity decreased. This change may directly affect glomerular and tubular cell function. In addition, sorbitol oxidizes fructose by coupling NAD + to generate NADH, which increases the proportion of intracellular $\mathrm{NADH} / \mathrm{NAD}+$, resulting in de novo synthesis of intracellular diacylglycerol (DAG) and activation of protein kinase $\mathrm{C}$ (PKC) Cause a series of biochemical and physiological changes. For the above 
biochemical metabolic abnormalities, for many years scholars have been committed to finding effective aldose reductase inhibitors to reduce polyol pathway metabolism, thereby reducing or blocking the occurrence of diabetic nephropathy.

\section{Protein Kinase C}

PKC widely exists in various tissues and cells of the human body and is a group of important protein kinases in cells. PKC can be activated by a variety of hormones, growth factors and neurotransmitters, phosphorylating many kinds of proteins in cells, constituting an important information network system in cells and regulating a series of physiological and biochemical functions of cells. The classic pathway of PKC activation is that after the cell receptor accepts the extracellular signal, it activates phospholipase C (PLC) and cleaves phosphatidylinositol diphosphate (PIP2) through G-protein coupling on the cell membrane to generate inositol triphosphate IP3) and diacylglycerol (DAG). IP3 Ca2 + Ca2 + release from the cyst or extracellular influx, so that intracellular Ca2 + content increased, in cooperation with DAG activation of PKC. Once activated, PKC produces a variety of short-term or long-term biological effects. Short-term effects include the release of hormones, neurotransmitters and various cytokines, the functioning of ion channels, the metabolism of nutrients, excitatory and contractile coupling of muscle cells, and immune and inflammatory responses. Long-term effects are mainly involved in the regulation of gene expression, protein synthesis and cell proliferation and differentiation. In the hyperglycemic state, glucose induces an increase in DAG synthesis, leading to an increase in intracellular DAG content, which in turn activates PKC. Activation of PKC, in turn, activates some intracellular transcription factors (C-fos, C-jun), which activate and enhance the transcriptional level of messenger RNA (mRNA) in extracellular matrix (fibronectin, laminin and collagen IV) increase. Activation of PKC also inhibits the activity of nitric oxide synthase, resulting in a decrease in NO production, which in turn inhibits the NO-mediated cGMP synthesis and results in altered vasoconstrictive activity. In the early stages of diabetic nephropathy in rats, specific PKC inhibitors can improve renal blood flow and glomerular filtration rate. These results indicate that hyperglycemia-activated PKC pathway directly or indirectly affects hemorheological changes. In addition, PKC regulates the adhesion, aggregation and secretion of platelets, stimulates the production of Von Willebrand factor in vascular endothelial cells and increases the content and activity of plasminogen activator inhibitor (PAI-1) in plasma or tissue, thereby promoting Diabetic vascular lesions hypercoagulable, low fibrinolysis and high blood viscosity formation. Phospholipase A2 (PLA2), an important phospholipase in the body, catalyzes the cleavage of phospholipids to produce free arachidonic acid. They are the precursors of prostaglandin, TXA2, platelet activating factor and other vasoactive substances and inflammatory mediators, which regulate and influence Vascular multiple physiological functions. Increased glomerular PLA2 activity in diabetic rats is also associated with activation of the PKC pathway. In addition, hyperglycemia activates intracellular PKC and PLA2 pathways, which are important mechanisms that lead to the decrease of $\mathrm{Na}+-\mathrm{K}+$ ATPas activity. PKC inhibitors restore normalized hypo-reduced $\mathrm{Na}+-\mathrm{K}+$ ATPas activity [1].

\section{Hemodynamic Changes}

The effects of diabetes on renal hemodynamics include two aspects: one is the effect of systemic hypertension; the other is the change of intrarenal hemodynamics. Renal hemodynamic changes directly involved in the occurrence of diabetic nephropathy, glomerular hyperfiltration plays a key role in which. Glomerular hyperfiltration can occur early in diabetes, with an increase in glomerular filtration rate (GFR) in $10 \%$ to $17 \%$ of early-stage diabetic patients. Micro-puncture technique for a variety of different types of diabetic nephropathy model of renal hemodynamic changes in the study results further confirmed that diabetic nephropathy GFR increase is mainly due to renal vascular resistance, especially the afferent arterioles and the arteriolar resistance Reduce, so that a single nephron plasma flow caused by increased [2]. Compared with the glomerular ascending aorta, the 
resistance of the arteriole into the ball decreased more obviously, so that glomerular filtration pressure increased, the phenomenon of hyperfiltration glomerular. In some normotensive diabetic rats using angiotensin-converting enzyme inhibitors and low-protein diet, no significant effect on systemic blood pressure, the glomerular can still significantly reduce the high pressure and high filtration state, with this Histological changes such as glomerular hypertrophy have also been alleviated. The above results show that glomerular capillary pressure is the leading cause of diabetic nephropathy tissue damage. It is also clinically observed that the occurrence of diabetic nephropathy and GFR increased significantly. A long-term follow-up of a group of patients with type I diabetes mellitus showed that in the early onset of GFR, the incidence of diabetic nephropathy was 53\% in 8 years, and the incidence of diabetic nephropathy the rate is only 5\%.

\section{Cytokine Effect}

Cytokines in the pathogenesis of diabetic nephropathy involves glomerular hemodynamic changes, extracellular matrix metabolism, cell proliferation and hypertrophy and many other aspects. According to the different functions of different cytokines, they can be summarized as cytokines, insulin-like growth factor (IGF-1) and platelet-derived growth factor (PDGF) involved in glomerular hemodynamic changes; There are TGF- $\beta$ and IGF-1, TGF- $\beta$, IGF- 1 and PDGF involved in the extracellular matrix metabolism; PDGF and fibroblast growth factor (FGF) involved in cell proliferation; Affect the insulin signaling are: tumor necrosis factor (TNF- $\alpha$ ) and IGF-1; involved in apoptosis are: TNF- $\alpha$ and TGF- $\beta 1$. In the case of diabetes, the above cytokine expression is regulated by insulin and blood glucose levels, AGE, PKC activity, hemodynamic changes, vasoactive factors, lipoprotein and protein intake and the like. Cytokines play a role through autocrine, paracrine, and endocrine pathways. Under the control of these factors, they interact and restrict each other to form a complex network of cytokines in the pathogenesis of diabetic nephropathy, in which TGF- $\beta 1$ is the core factor. Whether in the kidney of STZ-induced diabetic rats or in the kidneys of (BB) spontaneous diabetic rats and (NOD) spontaneously diabetic mice, TGF- $\beta 1 \mathrm{mRNA}$ expression and protein content were significantly increased with renal hypertrophy and extracellular matrix increased. After intervention with specific anti-TGF- $\beta 1$ antibody, renal hypertrophy and extracellular matrix production were significantly inhibited in diabetic rats. In addition, TGF- $\beta 1$ mRNA expression and its protein content were also significantly increased in renal biopsy tissues of patients with diabetic nephropathy. TGF- $\beta 1$ levels have begun to increase in kidney tissue in some patients with early-stage diabetes who have not yet developed diabetic nephropathy. The role of TGF- $\beta 1$ in the pathogenesis of diabetic nephropathy can be summarized as: to promote renal cell hypertrophy, increased mesangial cell extracellular matrix production, and by increasing the activity of matrix degrading enzyme inhibitors, inhibition of matrix-degrading enzyme synthesis and reduce extracellular Degradation of the matrix. In addition, TGF- $\beta 1$ also stimulates cells to synthesize extracellular matrix receptors (Integrins), triggering cell-to-cell and cell-to-extracellular matrix interactions [3].

\section{Gene Background}

The genetic background plays a very important role in the pathogenesis of diabetic nephropathy. In diabetic patients, not only diabetic nephropathy occurs in some patients, but also occurs in diabetic nephropathy. In some diabetic patients with a family history of hypertension, the incidence of diabetic nephropathy is also significantly higher than in patients without a family history of hypertension. In addition, there are also differences in the incidence of diabetic nephropathy among different ethnic groups. All these indicate that the occurrence of diabetic nephropathy is also related to genetic factors. Of course, environmental factors also play an important role in them. In individuals with predisposition to diabetes, obesity, age, and decreased physical activity speed up the development of diabetes. Diabetes is a polygenic disease [4]. The interaction between pathogenic genes and susceptible genes in the pathogenesis of diabetes mellitus and diabetic nephropathy constitutes the complexity of the genetic study of diabetic nephropathy [5]. 


\section{Conclusions}

Although the pathogenesis of $\mathrm{DN}$ is not yet clear, but from the current study should be oxidative stress, genetic factors, disorders of glucose and lipid metabolism, hemodynamic changes, vasoactive substances, a variety of cytokines such as excessive expression Factors related to further study of the pathogenesis of DN DN prevention and treatment will have an important guiding significance. In order to reduce the incidence of $\mathrm{DN}$, the search for modern Chinese medicine can significantly reduce the levels of CTGF, TGF- $\beta 1$ and type IV collagen in DN patients, reduce non-enzymatic glycation, inhibit renal fibrosis and protect renal function in DN patients, with clinical practical value [6].

\section{References}

[1] Zhang Zhenzhong, Dou Xiaoni, Zhao Hongbo clinical trial of Shenqi Tang Shenan Decoction in the treatment of diabetic nephropathy [J]. Journal of Shaanxi College of Traditional Chinese Medicine. 2007 (03):15-18.

[2] Zhang Zhenzhong, Dou Xiaoni, Zhao Hongbo, Ma Lijuan. Experimental study of Shenqi Tang Shenan capsule in the treatment of diabetic nephropathy [J]. Zhong Mei Zhong Yi Yao. 2007 (03):43-45.

[3] Li Zhaowei, Qu Liqing. Diabetic nephropathy syndrome differentiation [J]. Lizhen Guo Yi Guo. 2006 (03):14-19.

[4] Zhao Zongjiang. Professor Ye Chuanhui treatment of diabetic nephropathy ideas and methods [J]. China Integrative Medicine, 2006. (03):108-109.

[5] Li Xiaogui, Dong Zhenghua, Ding Hui etiology and pathogenesis of diabetic nephropathy [J]. Shanxi Traditional Chinese Medicine 2005 (06):77-83.

[6] Wei Chen, Zhao Zongjiang. The role of connective tissue growth factor in renal fibrosis [J]. China Integrative Medicine, 2005. (02):1351-1358. 